\title{
Diversidad, composición y estructura de un hábitat altamente amenazado: los bosques estacionalmente secos de Tarapoto, Perú
}

\section{Diversity, composition, and structure of a highly endangered habitat: the seasonally dry forests of Tarapoto, Peru}

\author{
Roosevelt García-Villacorta ${ }^{1,2,8}$
}

1Universidad Nacional de la Amazonía Peruana, Iquitos, Perú 2Departamento de Ecología y Biología Evolutiva, Universidad de Michigan, 830 North University, Aniversity, Ann Arbor, MI 48109-1048, USA, for Biodiversity \& Conservation (PCBC). E-mail: roosevelg@hotmail.com

Presentado: 01/09/2008 Aceptado: 21/04/2009 Publicado online: 28/08/2009

\section{Resumen}

Entre marzo y abril de 2006, la flora de los bosques estacionalmente secos en Tarapoto, San Martín fue estudiada en diez localidades. En cada una de estas localidades se estableció una parcela de 0,1 ha; cada tallo mayor de $2,5 \mathrm{~cm}$ de diámetro fue medido, contado e identificado a especie. Se encontraron 146 especies en 2814 individuos. En toda la zona de estudio, Myrtaceae fue la familia más diversa con 14 especies, seguida por Leguminosae con 12 especies. Igualmente, Annonaceae y Myrtaceae fueron las familias más abundantes en numero de tallos en toda la zona de estudio (461, y 412 tallos totales), mientras que Sapotaceae, con sólo 160 tallos totales, tuvo el valor más alto en área basal, debido a la Quinilla, Manilkara bidentata (A. DC.) A. Chev., el árbol más importante en la estructura de los bosques estacionalmente secos de Tarapoto. Dos especies estuvieron presentes en todos los sitios de estudio: Coccoloba sp. 1, (Polygonaceae) y Oxandra espintana (Spruce ex Benth.) Baill. (Annonaceae), mientras que 53 especies ocurrieron en un solo sitio. Los bosques estacionalmente secos de Tarapoto presentan una diversidad intermedia comparada con otros bosques secos del Neotrópico y similar diversidad a los bosques secos del Pacífico Peruano. La comunidad de árboles en estos bosques pertenece a especies de amplia distribución comparada con las especies de arbustos que tienen distribución restringida a los hábitats de bosques secos. En términos generales las áreas localizadas cerca de la carretera Tarapoto-Juanjui tienen el grado más alto de amenaza y perturbación debido a la extracción maderera y destrucción de hábitat para la creación de campos de cultivos agrícolas. Los bosques más representativos y mejor conservados de esta región se encuentran al Oeste y Sur de Picota, en el Área de Conservación Municipal El Quinillal, y en la cuenca del Río Bombonajillo y Ponasillo. Estos resultados resaltan la urgencia de conservar estos bosques ante la creciente amenaza de deforestación y pérdida de hábitat prevalente en la región.

Palabras clave: Área de Conservación Municipal, bosque estacionalmente seco, composición florística, diversidad, San Martín, Tarapoto.

\section{Abstract}

Between March and April 2006, I studied ten localities with seasonally dry forests located along the TarapotoJuanjui road, San Martin. At each site ten $50 \times 2 \mathrm{~m}$ transects totalling $1000 \mathrm{~m}^{2}(0,1 \mathrm{ha})$ were laid out along a 180 $\mathrm{m}$ baseline where all standing trees with diameter at breast height (DBH) greater than $2,5 \mathrm{~cm}$ were measured, counted and identified to species. I found a total of 146 species and 2814 individuals with $\mathrm{DBH} \geq 2,5 \mathrm{~cm}$. Within the study area, Myrtaceae was the most species rich family with 14 species followed closely by Leguminosae with 12 species. Annonaceae and Myrtaceae had the highest number of individuals (461 and 412, respectively), whereas Sapotaceae, with only 160 stems, had the highest basal area. The latter was mainly due to Manilkara bidentata (A. DC.) A. Chev. ("Quinilla"), the most important tree species in the seasonally dry forests of Tarapoto. Two species were present at all sites: Coccoloba sp. 1 (Polygonaceae) and Oxandra espintana (Spruce ex Benth.) (Annonaceae), whereas 53 species occurred only at one site. The seasonally dry forests of Tarapoto have an intermediate diversity compared to the other dry forests in the Neotropics and similar diversity compared to the Peruvian dry forests of the Pacific coast. Most of the tree community in these forests belong to geographically widespread species compared to shrubs that are mostly restricted to dry forests habitats. The areas located near the Tarapoto-Juanjui road ("carretera marginal") have the most disturbed forests. The best representations of Tarapoto's seasonally dry forests are located west and south of the locality of Picota, in the Area de Conservación Municipal "El Quinillal", and in the Bombonajillo and Ponasillo basins. These results highlight the pressing needs for conservation efforts in the area, before these unique forests are forever lost due to the continuing expansion of agricultural fields and logging activities prevalent in the region.

Keywords: Área de Conservación Municipal, seasonally dry forest, floristic composition, diversity, San Martín, Tarapoto.

\section{Introducción}

Los bosques secos Neotropicales se distribuyen en forma fragmentada desde México hasta el norte de Argentina. Prado y Gibbs (1993) presentaron la hipótesis del 'Arco pleistocénico' para sugerir que la naturaleza fragmentada en forma de arco de estos bosques en el presente sugieren la existencia en el pasado de bosques secos con distribución más continua y extensa, especialmente durante el Pleistoceno (18-12 Ma BP) y coincidente con la contracción de los bosques húmedos. Esta hipótesis es apoyada por un reciente estudio de genética poblacional en Astronium urundeuva (Allemão) Engl. (Anacardiaceae), un árbol restringido a los bosques estacionalmente secos del este de Sudamérica (Caetano et al. 2008).
Los bosques estacionalmente secos son más pequeños en estatura y menos complejos florística y estructuralmente que los bosques húmedos tropicales (Murphy y Lugo 1986). Cerca del $42 \%$ de los hábitats tropicales y subtropicales corresponden a bosques secos tropicales (Holdridge 1967), y en Sudamérica ellos representan el 22\% del área boscosa (Murphi y Lugo 1986). Miles et al. (2006) estimaron que más de la mitad de los bosques secos tropicales que quedan en el mundo (54,2\%) están localizados en Sudamérica. Lamentablemente, a lo largo del Neotrópico estos bosques están continuamente desapareciendo debido en parte a la ocupación de asentamientos humanos y el reemplazo de los bosques por campos agrícolas y pastos para ganadería (Maass 1995). Esta situación convierte a las comunidades de bosques secos en uno de los ecosistemas tropicales más amenazados del planeta (Janzen 1988, Maass 1995). 
La presencia de estos bosques esta aparentemente determinada por la cantidad, estacionalidad y distribución anual de las lluvias. Holdridge (1967) propuso que los bosques secos tropicales y subtropicales están localizados en áreas donde la biotemperatura anual es mayor a $17^{\circ} \mathrm{C}$, y el rango anual de precipitación está entre 250 a $2000 \mathrm{~mm}$. Murphy y Lugo (1986) sugirieron que sólo se necesitan entre dos a tres meses de estación seca para que la composición y estructura del bosque cambie de un bosque húmedo a un bosque seco tropical. Asimismo, Gentry (1995) usó el valor de $700-1600 \mathrm{~mm}$ de precipitación anual, con al menos una estación seca, como limite para definir los bosques secos en su análisis florístico de bosques secos Neotropicales. Estas diferencias en precipitación también son importantes en los tipos de bosques secos que existen en el Perú: por ejemplo cerca de $1020 \mathrm{~mm}$ en los bosques estacionalmente secos de Tarapoto, hasta un ambiente más árido en los bosques secos de Tumbes, que reciben un mínimo de $567 \mathrm{~mm}$ de lluvia anual (INRENA 1995).

ONERN (1976), basado en el trabajo de Holdridge (1947), usó bio-temperatura, precipitación y humedad ambiental para clasificar los bosques secos peruanos en dos tipos: bosques muy secos y bosques secos. Este último fue dividido en cinco subtipos: bosques secos tropicales, bosques secos tropicales pre-montanos, bosques secos sub-tropicales, bosques secos tropicales montanos bajos y bosques secos sub-tropicales montanos bajos. Los primeros dos subtipos fueron reportados para el departamento de San Martín (ONERN, 1976). En la misma línea, una clasificación más concisa de los bosques estacionalmente secos peruanos fue presentada por Linares-Palomino (2006): bosques tropicales estacionalmente secos ecuatoriales, bosques tropicales estacionalmente secos inter-Andinos y bosques tropicales estacionalmente secos del Este. Por otro lado, un análisis reciente muestra que los bosques estacionalmente secos de Tarapoto tienen una flora única, muy diferentes a los bosques estacionalmente secos interAndinos y del Pacifico (Linares-Palomino, 2006).

Los bosques estacionalmente secos de Tarapoto son particularmente interesantes por estar aislados de otros bosques secos peruanos, al Este de los Andes. Ellos ocupan las partes bajas y colinosas del bajo Río Mayo y Río Huallaga, en el área conocida como Huallaga central. De acuerdo al mapa ecológico del Perú, los bosques estacionalmente secos de Tarapoto son parte de un área más grande que incluye las localidades de Tarapoto, Bellavista y Juanjui. Bosques similares a los que ocurren en Tarapoto aparecen luego más al sur, en la confluencia de los Río Ene y Perené, y finalmente cerca de la localidad de Quillabamba (INRENA 1995).

La mayor parte de los esfuerzos para estudiar y conservar los bosques secos en el Perú han sido dedicados a los bosques de Tumbes y Piura (Linares-Palomino 2002, Leal-Pinedo y Linares-Palomino 2005, Linares-Palomino y Ponce Álvarez, 2005). Pocos estudios han sido realizados para mejorar el conocimiento de los bosques estacionalmente secos de Tarapoto, y los que se hicieron, estuvieron limitados a una sola localidad (Bridgewater et al. 2003, Phillips y Miller 2004). Aun peor es nuestro conocimiento sobre su estado de conservación considerando que una importante carretera, la Carretera Marginal de la Selva, cruza por entero el Huallaga Central. Este estudio tuvo como meta principal obtener información básica sobre la flora de los bosques estacionalmente secos de Tarapoto usando un protocolo de muestreo consistente que nos permitiera conocer su diversidad, estructura y endemismo. Así mismo se evaluó el estado de conservación de estos boques para sugerir donde focalizar los esfuerzos para conservar de este importante hábitat en el Noreste del Perú.

\section{Material y métodos}

\section{Lugares de estudio}

Las localidades que aun conservan remanentes bosques estacionalmente secos fueron seleccionadas usando una imagen de satélite Landsat TM (bandas 4, 5 y 7, fecha de toma 7 noviembre 1999, path=8, row=065) descargada del Global Land Cover Facility (http://glcf.umiacs.umd.edu/index.shtml). Para ubicar algunas localidades específicas se comparó la imagen con el mapa de vegetación de San Martín elaborado por el IIAP (Encarnación, 2004). Los lugares seleccionados se ubican en tres provincias del departamento de San Martín: San Martín, Picota y Bellavista. Para seleccionar los lugares específicos de muestreo se tuvo en consideración su accesibilidad y estado de conservación. Esto último se verificó por la ausencia de áreas perturbadas y campos de cultivo cercanos. Los suelos fueron predominantemente de color negro con piedras grandes y pequeńas dispersas en el terreno. El clima en la región de estudio tiene dos estaciones bien marcadas: húmeda (octubre-marzo) y seca (abril-setiembre). La precipitación media anual en Tarapoto es $1164,4 \mathrm{~mm}$ mientras que en Juanjui es 1433,3 mm. Hay poca variación en la temperatura en ambas localidades, con un valor medio de $26^{\circ} \mathrm{C}$.

\section{Inventario de Plantas}

En total se instalaron ocho parcelas de 0,1 ha $\left(1000 \mathrm{~m}^{2}\right)$, una de $500 \mathrm{~m}^{2}$ (Paucar) y otra de $700 \mathrm{~m}^{2}$ (Ledoy) para un total de 10 localidades muestreadas (Fig. 1). Todas las parcelas de estudio estuvieron localizadas tan lejos como fue posible de áreas de cultivo, bosques secundarios y pastos. Las parcelas fueron establecidas en laderas de colinas, sobre terrenos altamente disectados, con pendientes a veces alcanzando los $70^{\circ} \mathrm{o}$ más de inclinación. Cada parcela consistió de una línea base de $180 \mathrm{~m}$ en el cual 10 transectos de 50x $1 \mathrm{~m}$ fueron distribuidos perpendicularmente en la línea base y distanciados cada $20 \mathrm{~m}$ (Phillips y Miller, 2004). Cinco transectos estaban orientados hacia un lado de la línea base y cinco transectos hacia el otro lado, muestreando efectivamente $180 \times 100 \mathrm{~m}$ (1,8 ha) de bosque

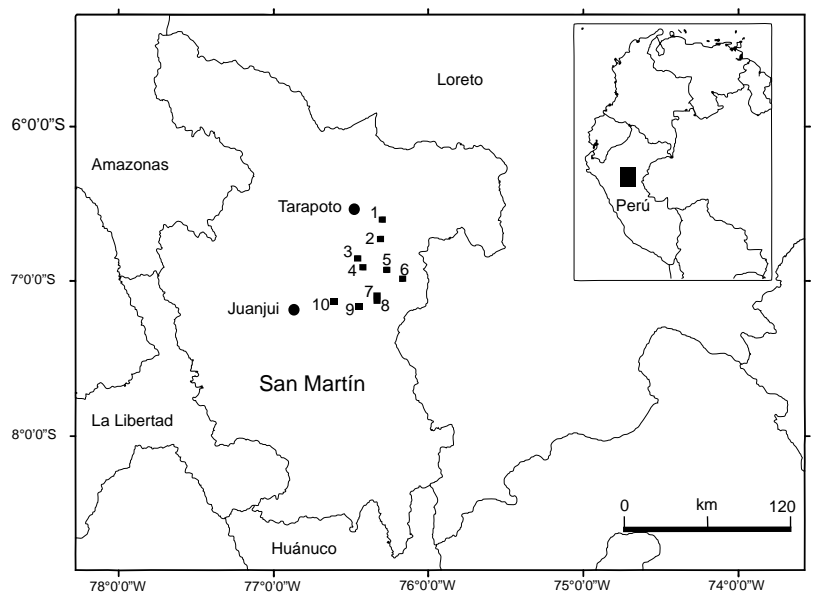

Figura 1. Mapa del departamento de San Martín y los sitios de estudio. (1) Juan Guerra, (2) Yacucatina, (3) Pucacaca, (4) Nueva Union, (5) Ponaza, (6) Paucar, (7) Bombonajillo, (8) Nuevo Control, (9) Biabo, (10) Ledoy. 
en cada sitio, excepto en los casos de parcelas más pequeñas. Todos los tallos más grandes de $2,5 \mathrm{~cm}$ de diámetro a la altura del pecho (DAP) fueron contados e identificados a especie o morfoespecie. Toda planta que no podía ser referida a un espécimen previamente muestreado fue colectado para su posterior identificación en el herbario.

Todas las plantas colectadas fueron preservadas en alcohol y secadas en las instalaciones del herbario USM del Museo de Historia Natural de la Universidad Nacional Mayor de San Marcos, Lima. Las colecciones de plantas fueron separadas en especies o morfoespecies para crear listados florísticos consistentes para cada parcela. Una copia completa de las colecciones (RG 41504650) está depositada en el herbario USM.

\section{Variación topográfica}

Para caracterizar la topografía de cada parcela, se registraron cambios en la pendiente cada $20 \mathrm{~m}$ a lo largo de la línea base utilizando un clinómetro Suunto ${ }^{\mathrm{TM}}$. Se midió también la pendiente dentro de los $20 \mathrm{~m}$ de intervalo cuando se encontró cambios substanciales en la pendiente. Paucar y Ledoy tuvieron menos puntos de medición debido al menor tamaño de las parcelas.

\section{Análisis de los Datos}

Para cada parcela calculé la densidad relativa, dominancia relativa, frecuencia relativa y el Índice de Valor de Importancia de especie (IVI)(Curtis y McIntosh 1951) usando las siguientes formulas:

$$
\begin{aligned}
& \text { Densidad Relativa: } \mathrm{DeR}_{j}=100 \times \mathrm{De}_{j} / \sum \mathrm{De}_{\mathrm{j}} \\
& \text { Dominancia Relativa: } \mathrm{DoR}_{j}=100 \times \mathrm{Do}_{\mathrm{j}} / \sum \mathrm{Do}_{\mathrm{j}} \\
& \text { Frecuencia Relativa: } \mathrm{FR}_{\mathrm{j}}=100 \times \mathrm{F}_{\mathrm{j}} / \sum \mathrm{F}_{\mathrm{j}}
\end{aligned}
$$$$
\text { Índice de Valor de Importancia }=\mathrm{IVI}_{\mathrm{j}}=\mathrm{DeR}_{\mathrm{j}}+\mathrm{DoR}_{\mathrm{j}}+\mathrm{FR}_{\mathrm{j}}
$$

Donde: $\mathrm{De}_{\mathrm{j}}$ es el número total de tallos de la especie $\mathrm{j}$ en todas las parcelas, Do es el área basal total de la especie $\mathrm{j}$ en todas parcelas, $\mathrm{F}_{\mathrm{j}}$ es el número de parcelas donde esta presenta la especie $\mathbf{j}$.

El porcentaje de similitud florística entre parcelas fue determinado calculando los coeficientes de similitud de Jaccard (usando datos de presencia/ausencia) y Bray-Curtis (usando datos de abundancia) (Magurran 1988).

Para representar visualmente las diferencias (o similitudes) de la flora entre sitios, se utilizó un análisis de agrupamiento y dos métodos de ordenación: Principal Coordinates Analysis (PCoA) y Non-metric Multidimensional Scaling (NMDS) (McCune y Grace 2002). En el análisis de agrupamiento se usó el índice de distancia de Bray-Curtis con datos de abundancia para calcular la similitud florística entre parcelas y el promedio de grupos (UPGMA) como método de enlace entre grupos. PCoA y NMDS son métodos de ordenación flexibles que permiten escoger el índice de similitud más apropiado en los cálculos. En este estudio usamos el índice de Bray-Curtis con datos de abundancia. NMDS representa en una matriz de dos dimensiones las distancias jerárquicas originales entre parcelas. Ambos métodos de ordenación representan juntas las parcelas que son florísticamente similares. El hecho que diferentes métodos de ordenación y análisis de agrupamiento resulten en el mismo patrón florístico reforzaría la conclusión que este patrón es real y no un artefacto del método usado.
Para estimar la eficacia del número de parcelas usadas en capturar la diversidad florística de los bosques secos de Tarapoto, se usaron las listas del inventario total para crear una curva de especies-área. También, los datos de abundancia en cada una de las parcelas fueron usados para crear una curva especies-distancia y estimar cuantas parcelas más se necesitarían para tener un listado consistente de especies a través de las parcelas. A diferencia de la curva especies-área que esta basado en datos de presencia/ ausencia, la curva especies-distancia usa datos de abundancia y proporciona una estimación del número de parcelas requeridas para tener una composición de especies consistente en toda el área de estudio.

Se determinó que familia y especie fue la más abundante y tuvo la mayor área basal en todo el inventario. Para investigar la estructura poblacional en estos bosques se calculó el área basal y número de tallos en cuatro clases diamétricas: $2,5-5,5-10$, $10-30, \geq 30 \mathrm{~cm}$. Estas clases diamétricas representan la estructura total del bosque desde juveniles hasta árboles adultos.

\section{Diversidad}

Los valores de diversidad por parcela fueron calculados de dos maneras: contando cuantas especies fueron encontrados en cada parcela (riqueza de especies) y calculando el valor Alfa de Fisher por parcela. Alfa de Fisher es un índice de diversidad que ha mostrado ser consistente a variaciones de abundancia comparado con otros índices de diversidad, y es cada vez más usado en comparaciones de diversidad florística entre parcelas (Condit et al. 1998, Phillips y Miller 2004).

Los métodos de ordenación, análisis de agrupamiento, similitud florística y cálculos del índice de Alfa de Fisher fueron realizados en el programa PAST v1.4 (http://folk.uio.no/ ohammer/past). PC-Ord v4.01 fue usado para crear la curva especies-área y la curva especies-distancia. Todos los otros análisis fueron ejecutados en Minitab ${ }^{\mathrm{TM}}$ y Excel $^{\mathrm{TM}}$.

\section{Estado de conservación de los bosques secos}

Para ayudar en la determinación del grado de conservación de los bosques estacionalmente secos inventariados, se asignó una categoría de amenaza de acuerdo al grado de perturbación observado en diez lugares de estudio: ligeramente amenazado (relativamente extensas áreas de bosques secos >1000 ha, carreteras distantes, cultivos esporádicos), medianamente amenazado (extensiones medianas de bosques secos $>100$ ha $y<1000$ ha, caminos o cultivos presentes), fuertemente amenazado (fragmentos de bosques secos $<100$ ha, grandes áreas degradadas, caminos y cultivos cercanos al área de estudio).

\section{Resultados}

\section{Patrones de diversidad}

Cientocuarentaiseis especies y morfoespecies fueron registradas en las diez localidades estudiadas (Apéndice 1). De estas, 75 fueron identificadas a nivel de especie, 40 a nivel de género, y 25 sólo pudieron ser identificadas a nivel de familia. Seis morfoespecies no fueron identificadas. Las morfoespecies registradas como indeterminados posiblemente representan extensiones de rango de especies reportadas en otros bosques secos, son generalistas de hábitat de bosques húmedos que llegan a extenderse hacia los bosques secos (e.g. Trichilia sp. 1, T. Pennington, com. pers.), o pueden representar nuevas especies para la ciencia (p. ejem. Croton sp. 1, Euphorbiaceae, P. Berry, 
Tabla 1, Características de la flora en 10 muestras (0,1 ha cada uno) de bosques estacionalmente secos de Tarapoto, San Martín. Nivel de Perturbación: 1 (ligeramente amenazado), 2 (medianamente amenazado), 3 (fuertemente amenazado), Paucar y Ledoy tuvieron 0,05 ha y 0,07 ha respectivamente.

\begin{tabular}{|c|c|c|c|c|c|c|}
\hline Sitio & Área basal $\left(\mathrm{cm}^{2}\right)$ & $\begin{array}{c}\text { Riqueza de } \\
\text { especies }\end{array}$ & Tallos por 0,1 ha & Alpha de Fisher & $\begin{array}{c}\text { Nivel de } \\
\text { perturbación }\end{array}$ & Elevación (m) \\
\hline Juan Guerra & 34203,14 & 50 & 280 & 17,72 & 2 & 191 \\
\hline Yacucatina & 24012,22 & 51 & 320 & 17,11 & 2 & 527 \\
\hline Pucacaca & 26638,54 & 56 & 371 & 18,32 & 2 & 322 \\
\hline Paucar & 8777,46 & 33 & 78 & 21,58 & 3 & 503 \\
\hline Nueva Unión & 21524,94 & 51 & 296 & 17,76 & 2 & 384 \\
\hline Biabo & 18593,33 & 50 & 380 & 15,41 & 1 & 393 \\
\hline Bombonajillo & 27736,10 & 43 & 283 & 14,11 & 1 & 304 \\
\hline Ledoy & 18700,42 & 39 & 194 & 14,70 & 2 & 273 \\
\hline Nuevo Control & 22835,60 & 45 & 329 & 14,10 & 3 & 284 \\
\hline Ponaza & 22094,05 & 51 & 283 & 18,16 & 2 & 260 \\
\hline
\end{tabular}

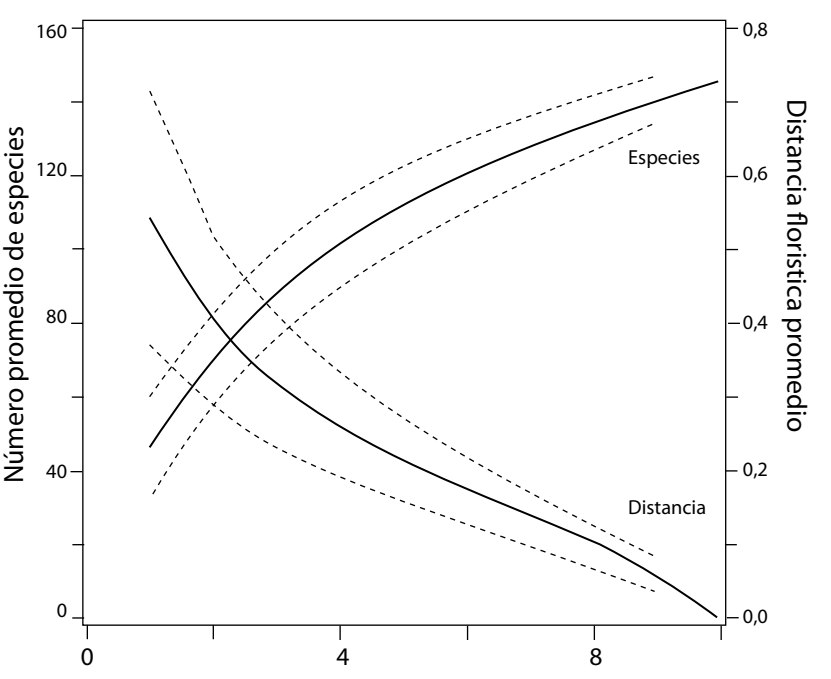

Figura 2. Curva especies-área y curva de distancia florística (BrayCurtis) entre las muestras y el total de especies encontradas en los bosques estacionalmente secos de Tarapoto. Las líneas con puntos representan bandas de confidencia.

com. pers.). La Tabla 1 muestra en resumen las características de las parcelas de estudio.

La curva especies-área muestra que este estudio capturó una gran proporción de la diversidad de especies en los bosques secos de Tarapoto (Fig. 2). La curva especies-distancia muestra que sólo cuatro a cinco parcelas de las dimensiones usadas en

Tabla 2. Diversidad por familia, área basal y abundancia de especies en los bosques estacionalmente secos de Tarapoto. En negrita las familias con mayor área basal.

\begin{tabular}{lccr}
\hline Familia & $\mathbf{N} .^{\circ}$ especies & $\mathbf{N}^{\circ}$ ind. & $\begin{array}{c}\text { Área basal } \\
\left(\mathbf{c m}^{\mathbf{2}} \mathbf{c}\right.\end{array}$ \\
\hline Myrtaceae & 14 & 412 & $\mathbf{1 8 6 2 1 , 8 3}$ \\
Fabaceae & 12 & 178 & $\mathbf{1 2 8 1 8 , 4 8}$ \\
Sapotaceae & 10 & 160 & $\mathbf{6 9 6 9 6 , \mathbf { 1 0 }}$ \\
Rubiaceae & 9 & 131 & 8723,83 \\
Annonaceae & 7 & 461 & $\mathbf{2 1 3 9 2 , 3 6}$ \\
Nyctaginaceae & 6 & 24 & 998,86 \\
Polygonaceae & 6 & 193 & 8308,07 \\
Apocynaceae & 5 & 16 & 3218,95 \\
Capparidaceae & 5 & 127 & 6286,97 \\
Euphorbiaceae & 5 & 105 & 7825,51 \\
Meliaceae & 5 & 172 & 11798,47 \\
Moraceae & 5 & 86 & $\mathbf{1 1 8 3 3 , 3 7}$ \\
Otras & 57 & 749 & 43593,00 \\
Total & 146 & 2814 & 225115,80 \\
\hline
\end{tabular}

este estudio son suficientes para capturar consistentemente la composición de especies en estos bosques.

Las cinco familias más importantes en número de especies en el inventario total fueron: Myrtaceae (14 especies), Leguminosae (12), Sapotaceae (10), Rubiaceae (9), y Annonaceae (7) (Tabla 2)

A nivel de género los cinco géneros con más especies en los bosques secos de Tarapoto son: Myrcia (5 especies, Myrtaceae), Pouteria (5, Sapotaceae), Trichilia (5, Meliaceae), Coccoloba (4, Polygonaceae), y Neea (4, Nyctaginaceae) (Tabla 3).

\section{Estructura del bosque}

El bosque en general tuvo baja altura oscilando entre $7-20$ $\mathrm{m}$, con algunos árboles emergentes alcanzando ca. $25 \mathrm{~m}$ (p.e. la palmera "inchawi”, Siagrus sancona H. Karst., Arecaceae). Casi la mitad de los tallos $(48,9 \%)$ estuvieron representados en la clase diamétrica más pequeña de $2,5-5 \mathrm{~cm}$ (Fig. 3a). La clase diamétrica más grande $(\geq 30 \mathrm{~cm})$ estuvo representado por sólo 50 tallos que corresponde a $1,7 \%$ del número total de tallos (Fig. 3a). La clase diamétrica de $10-30 \mathrm{~cm}$ fue la clase dominante en área basal con $43,2 \%$ del total, seguido por la clase diamétrica $\geq 30 \mathrm{~cm}$ con $34,6 \%$ (Fig. 3b).

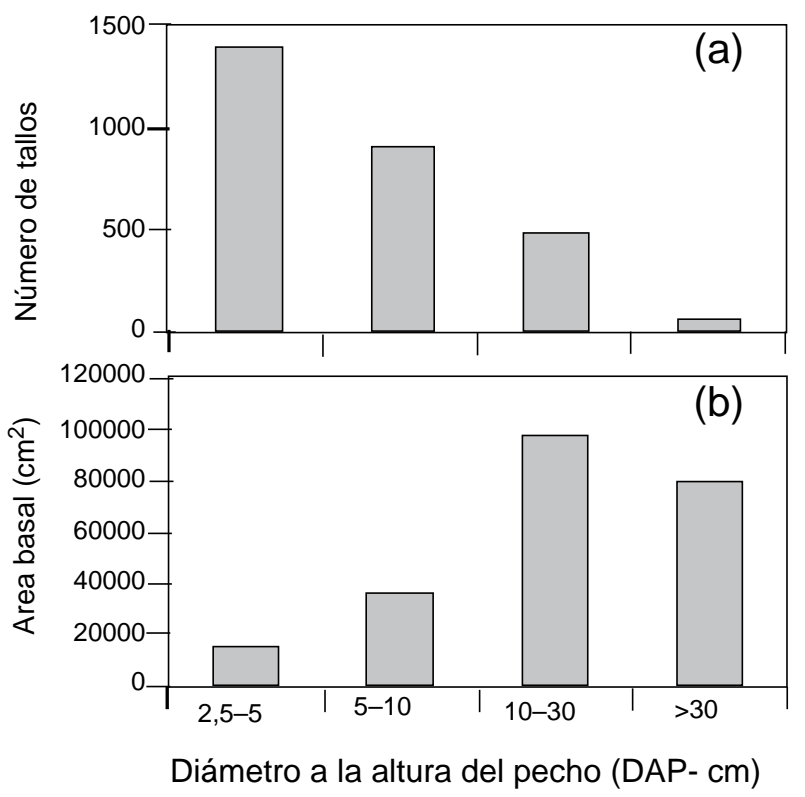

Figura 3. Estructura poblacional de los bosques estacionalmente secos de Tarapoto de acuerdo a área basal (a)(arriba) y número de tallos (b) (abajo)de acuerdo a cuatro clases diamétricas. 
Tabla 3. Los géneros más diversos en los bosques estacionalmente secos de Tarapoto, Familias abreviadas en paréntesis.

\begin{tabular}{lc}
\hline Género & No $^{\mathbf{0}}$ Especies \\
\hline Myrcia (Myrt) & 5 \\
Pouteria (Sapo) & 5 \\
Trichilia (Meli) & 5 \\
Coccoloba (Poly) & 4 \\
Neea (Nyct) & 4 \\
Brosimum (Mora) & 3 \\
Capparis (Capp) & 3 \\
Erythroxylum (Eryt) & 3 \\
Ocotea (Laur) & 3 \\
Tabebuia (Bign) & 3 \\
Allophylus (Sapi) & 2 \\
\hline
\end{tabular}

Biabo tuvo el más alto de número de tallos (380 tallos) mientras Juan Guerra, Ponaza y Bombonajillo tuvieron el número más bajo por parcela $(280,283$ y 283 respectivamente). El número promedio de tallos sin incluir Paucar y Ledoy fue 318 tallos. Juan Guerra, Bombonajillo y Pucacaca, en este orden, tuvieron el área basal más alto debido al número alto de tallos en la clase diamétrica $\geq 10 \mathrm{~cm}$ de diámetro (Tabla 4).

Más de la mitad de los árboles con DAP $\geq 30 \mathrm{~cm}(56 \%, 28$ tallos) pertenecieron a Manilkara bidentata (A. DC.) A. Chev. ("Quinilla", Sapotaceae), el cual al mismo tiempo tuvo el valor IVI más alto (Apéndice 1). La segunda especie más importante en esta clase diamétrica perteneció a una Myrtaceae (Myrcia sp. 3, "shucshumbo"), fuertemente explotada localmente para producir carbón (Bedmar García, Yacucatina, com. per.). El tallo con diámetro más grande en todo el inventario $(79,2 \mathrm{~cm}$ de DAP) perteneció también a un árbol de "Quinilla" (M. bidentata) seguido por especies menos comunes como Brosimum sp. 1 (Moraceae) y Schinopsis peruviana (Anacardiaceae), ambos con aproximadamente $50 \mathrm{~cm}$ de DAP. $17 \%$ de los árboles en la clase diamétrica de 10 - $30 \mathrm{~cm}$ DAP pertenecieron al árbol mediano Oxandra espintana (Spruce ex Benth.) Baill. ("espintana”, Annonaceae), seguido de nuevo por "Quinilla”, y Trichilia sp. 1

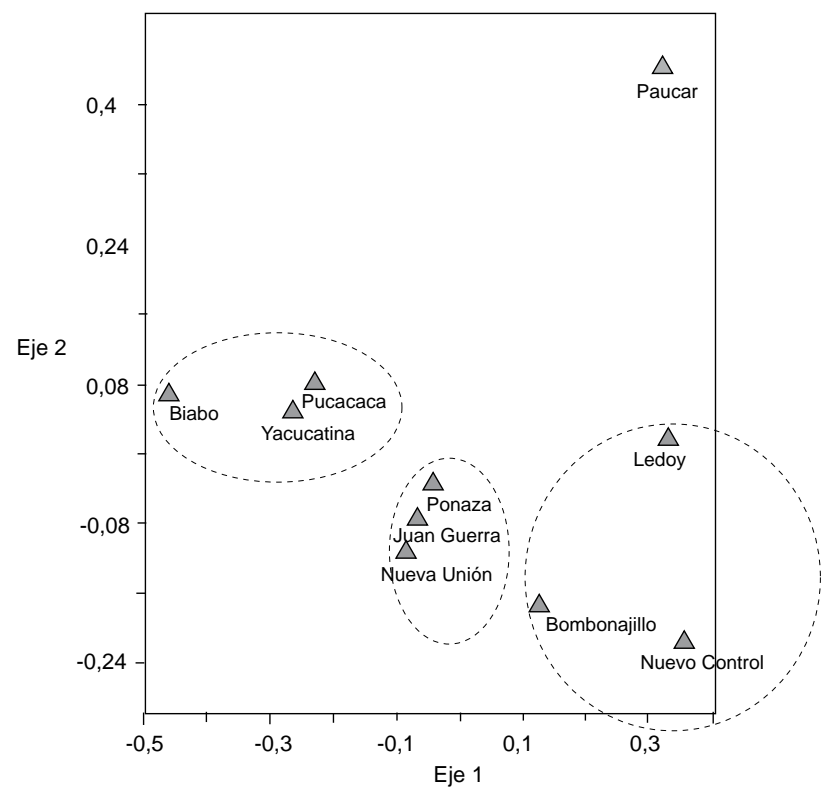

Figura 4. Non-metric multidimensional scaling (NMDS) de los sitios de estudio. Parcelas florísticamente similares están más juntos que parcelas florísticamente diferentes. ("uchumullaca", Meliaceae) con 5,9\% y 5,7\% del número total de tallos respectivamente.

Casi un décimo (10,7\%) del tamaño de clase entre 5 a 10 cm DAP perteneció a O. espintana (Annonaceae) seguido por Maytenus macrocarpa (Ruiz \& Pav.) Briq. ("chuchuhuasi”, Celastraceae), Coccoloba sp. 3 (Polygonaceae) y Gustavia elliptica S.A. Mori ("chopé", Lecythidaceae) con 5,3\%, 5,3\% y 4,2\% respectivamente. Las especie de sotobosque localmente conocido como "chopé" (G. elliptica) fue inusualmente abundante en la parcela Nuevo Control donde representó 15\% del total de área basal y 26\% del número total de tallos. De hecho, del total de 96 individuos registrados en toda el área de estudio, 86 individuos (90\%) fueron registrados solamente en esta parcela.

En el tamaño de clase más pequeño de $2,5-5 \mathrm{~cm}$ de DAP, cuatro especies representaron más del $30 \%$ del número total de tallos: Myrcia sp. 1 (9,5\%, 131 tallos, (Myrtaceae), O. espintana (8,3\%, 115 tallos, Annonaceae), Xylopia cuspidata (6,6\%, 91 tallos, Annonaceae) y Myrcia sp. 4 (5,7\%, 78 tallos, Myrtaceae). La tabla 4 muestra las diez especies más abundantes en cada parcela de estudio.

\section{Composición florística}

Tanto el PCoA como el análisis NMDS mostraron los mismos patrones de relaciones entre parcelas y sólo se presentan los resultados del análisis NMDS (Figs. 4). En ambos análisis la parcela de Paucar se comportó como una parcela atípica, sin agruparse consistentemente con las otras parcelas. Dos razones me hacen hipotetizar que este comportamiento atípico no es debido al menor tamaño de las parcelas. Primero, la parcela en Ledoy también tuvo un tamaño menor al de las otras parcelas de estudio y sin embargo la composición encontrada fue suficiente para que esta parcela se agrupe con uno de los grupos florísticos encontrados en toda el área de estudio. Segundo, durante el trabajo de campo en Paucar fue evidente que la composición

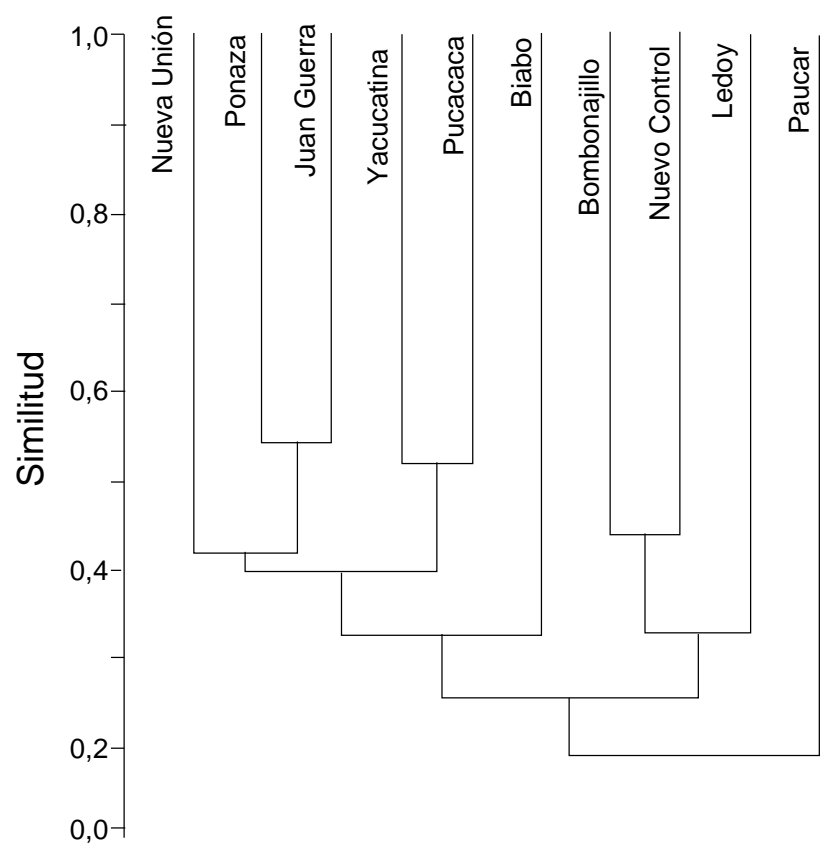

Figura 5. Análisis de agrupamiento basado en la similitud entre parcelas (índice Bray-Curtis). Similitud entre parcelas varía desde cero (parcelas complemente diferentes) hasta uno (parcelas completamente similares). 
Tabla 4. Las 10 especies más abundantes en cada muestra de bosque estacionalmente seco de Tarapoto estudiado.

\begin{tabular}{|c|c|c|c|c|c|}
\hline Sitio & Especies & $\begin{array}{c}\text { N.o de } \\
\text { Tallos } \\
\text { por } 0,1 \\
\text { Ha }\end{array}$ & Sitio & Especies & $\begin{array}{c}\text { N.o de } \\
\text { Tallos } \\
\text { por } 0,1 \\
\text { Ha }\end{array}$ \\
\hline \multirow{10}{*}{ 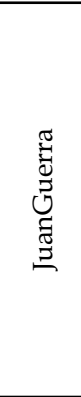 } & Trichilia sp. 1 & 26 & \multirow{10}{*}{$\frac{8}{. \pi}$} & Myrcia sp. 1 & 120 \\
\hline & Oxandra espintana (Spruce ex Benth.) Baill. & 25 & & Oxandra espintana (Spruce ex Benth.) Baill. & 43 \\
\hline & Myrcia sp. 2 & 18 & & Erythroxylum lucidum Kunth & 43 \\
\hline & Manilkara bidentata (A. DC.) A. Chev. & 16 & & Simira rubescens $c f$. & 16 \\
\hline & Drypetes amazonica Steyerm. & 14 & & Cordia alliodora (Ruiz \& Pav.) Oken & 13 \\
\hline & Brosimum sp. 1 & 13 & & Alseis peruviana Standl. & 12 \\
\hline & Allophylus loretensis cf. & 12 & & Arecaceae sp. 1 & 11 \\
\hline & Erythroxylum shatona J.F. Macbr. & 9 & & Indet. 4 & 10 \\
\hline & Capparis petiolaris Kunth & 9 & & Fabaceae sp. 1 & 9 \\
\hline & Capparis mollis Kunth & 9 & & Brosimum sp. 1 & 6 \\
\hline \multirow{10}{*}{ 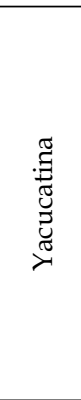 } & Oxandra espintana (Spruce ex Benth.) Baill. & 65 & \multirow{10}{*}{ 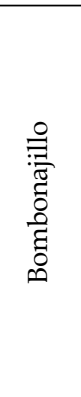 } & Zygia coccinea aff. & 42 \\
\hline & Maytenus macrocarpa (Ruiz \& Pav.) Briq. & 33 & & Myrcia sp. 4 & 37 \\
\hline & Myrtaceae sp. 6 & 26 & & Triplaris americana $\mathrm{L}$. & 25 \\
\hline & Coccoloba sp. 3 & 24 & & Capparis petiolaris Kunth & 23 \\
\hline & Trichilia rubra C. DC. & 15 & & Trichilia sp. 1 & 16 \\
\hline & Simira rubescens $c f$. & 9 & & Oxandra espintana (Spruce ex Benth.) Baill. & 11 \\
\hline & Manilkara bidentata A. DC.) A. Chev. & 9 & & Allophylus loretensis cf. & 11 \\
\hline & Platymiscium sp. 1 & 9 & & Trichilia maynasiana C. DC. ssp. maynasiana & 10 \\
\hline & Erythroxylum lucidum Kunth & 9 & & Manilkara bidentata (A. DC.) A. Chev. & 9 \\
\hline & Drypetes amazonica Steyerm. & 8 & & Brosimum sp. 1 & 8 \\
\hline \multirow{10}{*}{$\begin{array}{l}\tilde{J} \\
\mathbb{Z} \\
\mathbb{Z} \\
\Xi\end{array}$} & Oxandra espintana (Spruce ex Benth.) Baill. & 78 & \multirow{10}{*}{$\frac{\overrightarrow{0}}{0}$} & Pouteria sp. 1 & 21 \\
\hline & Indet. 4 & 27 & & Sorocea trophoides W.C. Burger & 16 \\
\hline & Maytenus macrocarpa (Ruiz \& Pav.) Briq. & 23 & & Oxandra espintana (Spruce ex Benth.) Baill. & 14 \\
\hline & Cordia alliodora (Ruiz \& Pav.) Oken & 21 & & Xylopia cuspidate Diels & 13 \\
\hline & Myrcia sp. 4 & 20 & & Protium puncticulatum J.F. Macbr. & 12 \\
\hline & Simira rubescens $c f$. & 17 & & Trichilia sp. 1 & 10 \\
\hline & Capparis mollis Kunth & 16 & & Croton glabellus L. & 10 \\
\hline & Capparis petiolaris Kunth & 16 & & Randia armata (Sw.) DC. & 10 \\
\hline & Myrcia sp. 3 & 16 & & Ocotea bofo Kunth & 10 \\
\hline & Mosannona Raimondi (Diels) Chatrou & 12 & & Pouteria sp. 2 & 9 \\
\hline \multirow{10}{*}{$\begin{array}{l}\overline{\widetilde{J}} \\
\bar{\Xi} \\
\text { c }\end{array}$} & Oxandra espintana (Spruce ex Benth.) Baill. & 11 & \multirow{10}{*}{ 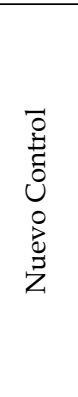 } & Gustavia elliptica cf. & 86 \\
\hline & Mosannona Raimondi (Diels) Chatrou & 10 & & Xylopia cuspidata Diels & 48 \\
\hline & Crematosperma sp. 1 & 6 & & Myrcia sp. 4 & 23 \\
\hline & Xylopia cuspidata Diels & 5 & & Trichilia maynasiana C. DC. ssp. Maynasiana & 18 \\
\hline & Pouteria subrotata cf. & 5 & & Pouteria sp. 1 & 14 \\
\hline & Fabaceae sp. 1 & 4 & & Mosannona Raimondi (Diels) Chatrou & 12 \\
\hline & Pouteria sp. 1 & 4 & & Pouteria sp. 2 & 12 \\
\hline & Protium puncticulatum J.F. Macbr. & 3 & & Zygia coccinea aff. & 11 \\
\hline & Clavija sp. 1 & 3 & & Coccoloba sp. 2 & 10 \\
\hline & Cordia alliodora (Ruiz \& Pav.) Oken & 2 & & Trichilia sp. 1 & 8 \\
\hline \multirow{10}{*}{ 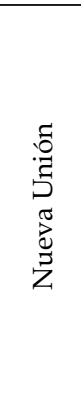 } & Myrcia sp. 1 & 25 & \multirow{10}{*}{$\begin{array}{l}\widetilde{J} \\
\text { ̋ี } \\
0\end{array}$} & Oxandra espintana (Spruce ex Benth.) Baill. & 25 \\
\hline & Coccoloba sp. 3 & 23 & & Maytenus macrocarpa (Ruiz \& Pav.) Briq. & 21 \\
\hline & Oxandra espintana (Spruce ex Benth.) Baill. & 20 & & Coccoloba sp. 3 & 20 \\
\hline & Zygia coccinea aff. & 20 & & Drypetes amazonica Steyerm. & 17 \\
\hline & Myrcia sp. 5 & 19 & & Xylopia cuspidata Diels & 16 \\
\hline & Ocotea sp. 1 & 15 & & Manilkara bidentata (A. DC.) A. Chev. & 16 \\
\hline & Croton sp. 1 & 13 & & Trichilia rubra C. DC. & 11 \\
\hline & Allophylus loretensis cf. & 11 & & Trichilia sp. 1 & 10 \\
\hline & Simira rubescens $c f$. & 10 & & Erythroxylum shatona J.F. Macbr. & 10 \\
\hline & Trichilia sp. 1 & 10 & & Allophylus loretensis $c f$. & 9 \\
\hline
\end{tabular}

de su flora era más similar a la que se encuentran en los bosques más húmedos del Sur de San Martín y posiblemente reflejen su cercanía a estos hábitats.

Además de Paucar otros tres grupos fueron evidentes en ambos diagramas: 1) Biabo, Pucacaca y Yacucatina, 2) Ponaza, Juan Guerra y Nueva Unión, 3) Ledoy, Bombonajillo y Nuevo Control. Estos grupos de parcelas comparten una flora más

similar entre si que con los otros grupos. Este mismo patrón fue evidente en el diagrama del análisis de agrupamiento (Fig. 5).

El porcentaje de similitud entre parcelas varió entre 17\% a $49 \%$ usando datos de presencia/ausencia y entre $9 \%$ a $53 \%$ usando datos de abundancia (Tabla 5). En general el porcentaje de similitud entre parcelas se incrementa cuando se incluyeron datos de abundancia. Por ejemplo, la similitud florística entre 
Tabla 5, Similitud florística entre pares de parcelas estudiadas, Parte superior derecha basado en el coeficiente de similitud Jaccard con datos de presencia/ausencia, Parte inferior izquierda basado en el coeficiente de similitud Bray-Curtis con datos de abundancia, JG= Juan Guerra, YA= Yacucatina, $P U=$ Pucacaca, $P A=$ Paucar, NU= Nueva Unión, $\mathrm{BI}=\mathrm{Biabo}, \mathrm{BO}=$ Bombonajillo, $\mathrm{LE}=$ Ledoy, $\mathrm{NC}=$ Nuevo Control, $\mathrm{PO}=$ Ponaza,

\begin{tabular}{|c|cccccccccc}
\hline & JG & YA & PU & PA & NU & BI & BO & LE & NC & PO \\
\hline JG & & 0,31 & 0,28 & 0,17 & 0,33 & 0,37 & 0,33 & 0,33 & 0,27 & 0,38 \\
YA & 0,36 & & 0,32 & 0,18 & 0,36 & 0,36 & 0,24 & 0,23 & 0,20 & 0,36 \\
PU & 0,36 & 0,50 & & 0,17 & 0,34 & 0,29 & 0,27 & 0,23 & 0,23 & 0,41 \\
PA & 0,16 & 0,14 & 0,17 & & 0,24 & 0,19 & 0,23 & 0,22 & 0,22 & 0,29 \\
NU & 0,40 & 0,39 & 0,32 & 0,18 & & 0,31 & 0,42 & 0,32 & 0,37 & 0,38 \\
BI & 0,30 & 0,34 & 0,34 & 0,12 & 0,32 & & 0,26 & 0,31 & 0,25 & 0,40 \\
BO & 0,39 & 0,19 & 0,28 & 0,18 & 0,40 & 0,14 & & 0,32 & 0,49 & 0,42 \\
LE & 0,32 & 0,19 & 0,19 & 0,24 & 0,27 & 0,18 & 0,30 & & 0,33 & 0,32 \\
NC & 0,23 & 0,12 & 0,21 & 0,18 & 0,28 & 0,09 & 0,43 & 0,35 & & 0,35 \\
PO & 0,53 & 0,50 & 0,38 & 0,20 & 0,42 & 0,27 & 0,40 & 0,35 & 0,27 & \\
\hline
\end{tabular}

Pucacaca y Yacucatina fue 32\% cuando se usó datos de presencia/ausencia (coeficiente de Jaccard) mientras que este valor se incrementó a 50\% cuando se incluyó en el análisis la abundancia de cada especie en las parcelas (coeficiente Bray-Curtis). Casos similares ocurrieron con los valores de Ponaza vs. Juan Guerra (de 38 a 53\%), Ponaza vs. Yacucatina (de 36 a 50\%) y Pucacaca vs. Yacucatina (de 32 a 50\%). La parcela de Paucar tuvo el valor más bajo de similitud florística con respecto a otras parcelas. Este valor varió entre $17-29 \%$ con datos de presencia/ausencia y $12-24 \%$ con datos de abundancia (Tabla 5). La figura 5 muestra una representación gráfica de las similitudes florísticas usando el índice de Bray-Curtis.

\section{Discusión}

Este estudio actualiza el número de especies leñosas reportadas por Linares-Palomino (2006) para Tarapoto de 108 a 146 especies. Usando el mismo protocolo de muestreo que el usado en este estudio, A. Gentry reportó que los bosques secos de los Cerros de Amotape tenían 43 especies (Phillips y Miller 2004), un poco menos que las 50 especies en promedio encontradas en Tarapoto. Una comparación de la diversidad de especies en muestras de 0,1 ha de bosques secos del Neotrópico muestra que la región de Tarapoto tiene una diversidad intermedia. Así por ejemplo la parcela en Bombonajillo con 43 especies es similar a los bosques secos del Cerro de Amotape en la región del Pacífico Peruano (Phillips y Miller 2002). Bosques más pobres en número de especies en tamańo de muestra similar ocurren en Calipán, México, con sólo 22 especies (Trejo y Dirzo 2002). Comparado con otras 60 muestras de bosques secos del Neotrópico las parcelas de Yacucatina, Nueva Unión y Ponaza (con 39, 40 y 41 especies respectivamente) tienen el número promedio de especies que se espera encontrar en muestras de 0,1 ha (R. García, datos no publicados). Los bosques secos más diversos en muestras similares se encuentran en Bolivia, en la zona de Nuevo Mundo y Río Negro, con 69 y 70 especies respectivamente (Phillips y Miller 2002).

El índice Alfa de Fisher varió de 14,1 (Nuevo Control y Bombonajillo) y 21,6 (Paucar). El valor relativamente alto del índice de Fisher en Paucar es notable si se tiene en cuenta el tamaño del muestreo (0,05 ha). El índice Alfa de Fisher es un índice de diversidad consistente (aun en comparaciones de muestras con número diferente de tallos y tamaño de parcela, Condit et al. 1998). Así, el mayor valor de Paucar puede probablemente reflejar su alta diversidad comparada a las otras parcelas (Tabla 1). Es de mencionar que la parcela en Paucar tenía elementos florísticos ligeramente diferentes de las otras muestras de bosques secos en Tarapoto y podría representar un bosque en transición de bosque seco a uno más húmedo con mayor diversidad. Pucacaca y Ponaza siguieron a Paucar en los valores altos de Alfa de Fisher con 18,32 y 18,16 respectivamente. La riqueza de especies por parcela (excluyendo a Paucar y Ledoy que tuvieron muestras de diferente tamaño) tuvo el valor más alto en Pucacaca (56 spp.) y más bajo en Bombonajillo (43 spp.).

En su única muestra $(0,1$ ha) de los bosques estacionalmente secos de Tarapoto, Gentry (1995) encontró que Myrtaceae (7 spp.) fue la tercera familia más importante en número de especies, después de Leguminosae y Rubiaceae. El presente estudio coloca Myrtaceae a la cabeza en número de especies en toda la zona estudiada, ocupando la primera posición en número de especies en Juan Guerra, Pucacaca y Nueva Unión, y la segunda posición en Ledoy, Biabo y Yucacatina. El género Myrcia es de resaltar ya que tuvo tres especies diferentes a la cabeza de las diez especies con el valor más alto de IVI en toda la región estudiada (Apéndice 1). En Santo Tomas (Colombia) y los bosques secos de Esmeraldas y Perro Muerto, Pacífico ecuatoriano, Myrtaceae también ocupa una posición prominente (Phillips y Miller 2004).

Ninguno de los géneros más diversos en el área estudiada (Myrcia, Pouteria, Trichilia, Coccoloba, Neea) está restringido a los ambientes de bosques secos sino que son ampliamente distribuidos en el Neotrópico. Este resultado concuerda con un análisis florístico previo de 28 muestras de bosques secos, donde se encontró que más de la mitad de ca. 350 géneros de plantas leñosas que ocurren en los bosques secos del Neotrópico estuvieron también distribuidos en los bosques húmedos (Gentry, 1995). Hay que resaltar sin embargo que otros géneros, especialmente arbustivos y herbáceos, contienen especies restringidos o presentan inusual diversidad en los bosques estacionalmente secos de Tarapoto (ver más abajo).

La evaluación completa del número total de especies endémicas no es posible en este trabajo ya que casi la mitad de las especies identificadas solo pudieron determinarse a nivel de género. Esto fue en parte una consecuencia inevitable de colectar especimenes estériles. Más trabajo de revisión en otros herbarios o colección de especimenes fértiles pueden mejorar nuestro conocimiento del rango de distribución las especies en los bosques secos de Tarapoto. A pesar de esta dificultad algunos de las especies más importantes en el área de estudio fueron completamente identificados. Para determinar su grado de endemismo se compiló la distribución geográfica de estas especies mediante la búsqueda en diversas fuentes que incluyó Tropicos, (Missouri Botanical Garden, www.mobot.org) y la base de datos de New York Botanical Garden.

El helecho epífito Platycerium andinum Baker es el único representante del género Platycerium que se encuentra en las Américas. Esta especie fue la más común en bosques secos con abundancia de árboles de "Quinilla" (M. bidentata, Sapotaceae). El patrón de ramificación amplio de estos árboles parece convertirlo en un hospedero perfecto para el establecimiento y crecimiento de individuos de esta especie de helecho. $P$. andinum sólo se encuentra en Perú en los bosques estacionalmente 
secos de Tarapoto y un poco mas al Sur en el Parque Nacional Cordillera Azul y bosques secos de Junín y Puno (Foster et al. 2001, Fernández y Vail 2003). El único otro reporte en Sudamérica de esta especie viene de colecciones aisladas en parches de bosques secos de la región de Madidi en Bolivia (Fernández y Vail 2003).

Desde una perspectiva de conservación, la concentración de especies endémicas en Tarapoto puede ser más importante que la presencia de especies generalistas de amplia distribución. Una observación general del listado de especies en nuestra área de estudio sugiere que la mayor parte de las especies restringidas a los bosques estacionalmente secos de Tarapoto son mayormente arbustos. Gentry (1995) comentó acerca de la conspicua representación de Erythroxylaceae y Capparaceae, dos familias mayormente de arbustos, en los bosques estacionalmente secos del Neotrópico. En nuestra área de estudio el género Erythroxylum (Erythroxylaceae) parece ser especialmente importante ya que Erythroxylum shatona J.F. Macbr. es endémico de los bosques secos de los departamentos de Amazonas y San Martín (León \& Monsalve 2006), y Erythroxylum lucidum Kunth se conoce en el Perú de Loreto y San Martín, así mismo el arbusto Steriphoma peruvianum Spruce ex Eichler (Capparaceae) se encuentra sólo en los bosques secos de Amazonas y San Martín.

Sin embargo la mayor parte de los árboles encontrados en las parcelas de estudio son generalistas distribuidos ampliamente en el Neotrópico (e.g. Manilkara bidentata, Maytenus macrocarpa (Ruiz \& Pav.) Briq., Drypetes amazonica Steyerm.), con unas pocas excepciones, como el árbol espinoso Schinopsis peruviana Engl. (Anacardiaceae), una especie restricta a bosques secos en Perú. Estos resultados hacen imperativo que estudios futuros en la zona incluyan diferentes épocas del año a fin de colectar los especímenes en condición fértil para facilitar su identifica- ción taxonómica. También será necesario colectar con mayor intensidad la flora de hierbas y arbustos donde posiblemente se encuentren la mayoría de las especies restringidas a los bosques estacionalmente secos de Tarapoto.

En los análisis de ordenación y agrupamiento fue posible definir tres grupos con flora similar (Figs. 4 y 5 ) que podría estar relacionado a patrones climatológicos prevalentes en el área. Los valles y montańas del área estudiada están variadamente localizados más cercanos o alejados de las fuentes de agua, donde el Río Huallaga es el más importante, pero teniendo otros como el Río Biabo, Bombonajillo y Ponasillo hacia el Sureste de Tarapoto. Estas diferencias en distancias de las fuentes de agua pueden influenciar el microclima de los valles del Huallaga Central y por ende el movimiento de los dispersores de semillas a través de los bosques secos. La abundancia de algunas especies como Myrcia sp. 3 ("shucshumbo") por ejemplo parece ser más importante en sitios ubicados al norte de Nueva Unión (Juan Guerra, Yacucatina, Pucacaca), y el árbol endémico $S$. peruviana (Anacardiaceae) es más abundante en Juan Guerra y Yucacatina. Es posible, que la manera en que las parcelas están agrupadas en el análisis de ordenación sea un reflejo de cambios en la humedad o precipitación, desde un clima relativamente más seco en Tarapoto a un clima más húmedo en Juanjui.

\section{Estado de conservación de los bosques estacionalmente secos de Tarapoto, San Martín}

Los bosques estacionalmente secos de Tarapoto están continuamente desapareciendo debido a la expansión agrícola. En su presente estado corresponde a una matriz de campos agrícolas, bosques secundarios, pastos y remanentes de bosques secos. Estos remanentes de bosques secos están principalmente localizados en las laderas de colinas inclinadas donde actividades económicas son difíciles de llevar a cabo (Fig. 6).

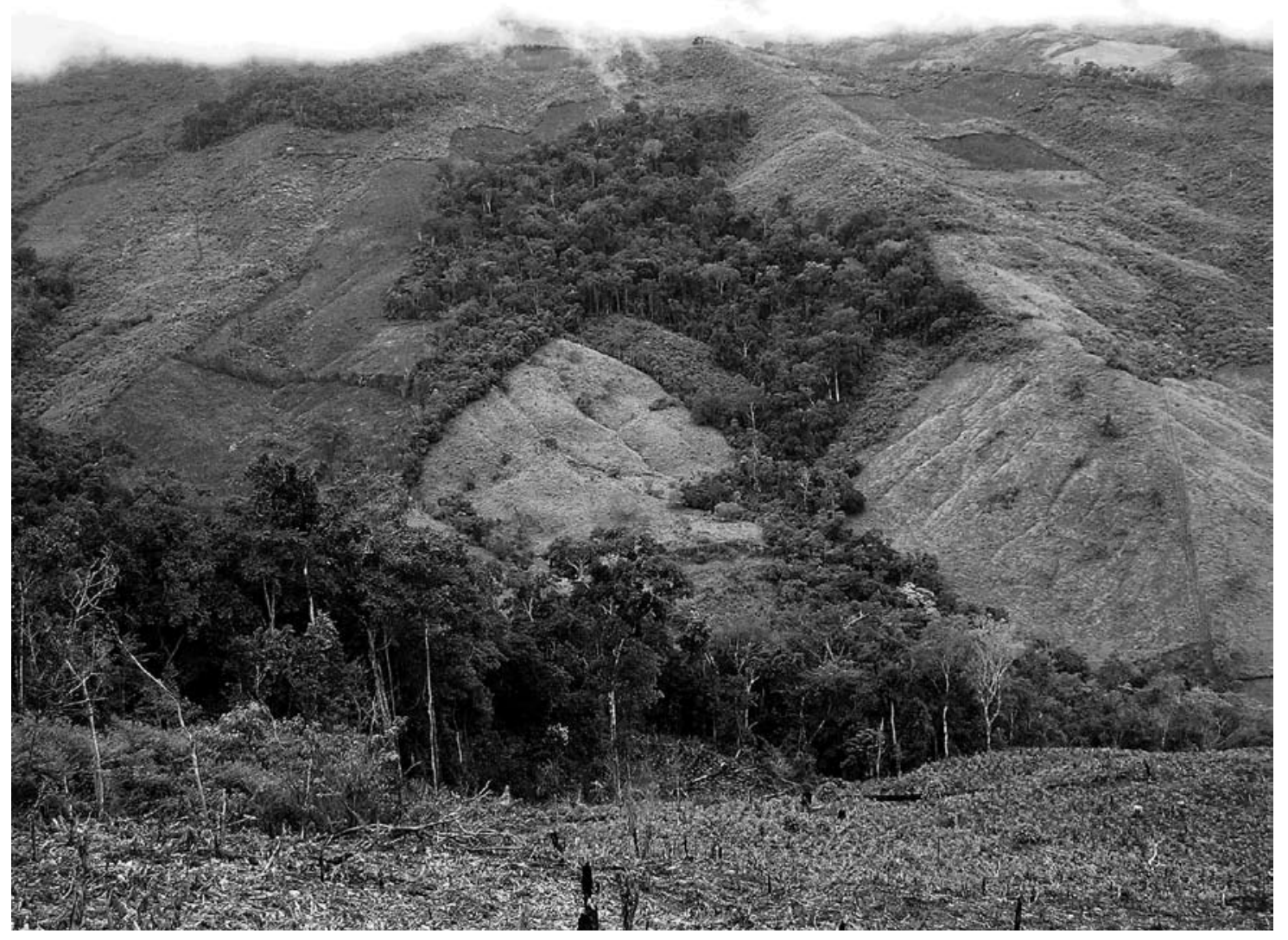

Figura 6.

Los bosques estacionalmente secos en Tarapoto están fuertemente fragmentados por actividades agrícolas. Los remanentes de bosques se encuentran en las partes más inclinadas de las colinas. 
El departamento de San Martín, y especialmente el valle del Huallaga Central, ha sido visto históricamente como una de las principales áreas para el desarrollo en gran escala de la expansión agrícola en el Perú. Esta visión fue alentada por la creación de la carretera Marginal de la Selva que atraviesa casi toda la extensión de hábitat de bosques estacionalmente secos en San Martín. La conservación de estos bosques es un complemento necesario si nuestra meta es preservar una parte representativa de la biodiversidad que existe en el Perú. Por esta razón es importante resaltar la labor realizada hasta el momento por algunos gobiernos locales en San Martín con la creación de 32 iniciativas de conservación municipal. Dos de estas áreas incluyen bosques estacionalmente secos: El Área de Conservación Municipal (ACM) El Quinillal y el ACM Pucararca-Ledoy. Estas áreas, especialmente el ACM El Quinillal, deben ser el punto de partida para la creación de áreas de conservación con categorías más estables y con mayor participación del gobierno regional y el INRENA, que sirvan para disuadir la tala ilegal y la invasión de tierras, procesos a los que estas ACM están actualmente expuestas.

Reportes previos han sugerido que los bosques estacionalmente secos más representativos están localizados a lo largo de la carretera Bellavista-Juanjui (Linares-Palomino, 2007). Este estudio por el contrario encontró que estas zonas presentan bosques mayormente degradados o destinadas a cultivos, especialmente de arroz (Ministerio de Agricultura 2006). De hecho, el muestreo de esta zona tuvo que obviarse debido a que no existían bosques secos en buen estado. Así mismo, Bellavista es uno de las provincias de San Martín con la tasa más alta de deforestación (Ministerio de Agricultura, 2006). Junto con los bosques de la cuenca del río Bombonajillo y el Área de Conservación Municipal El Quinillal, las áreas mejor preservadas y representativas de los bosques secos en la región San Martín fueron encontrados al este de Juanjui, en áreas cerca de Nueva Unión y al Sureste de Ledoy.

\section{Recomendaciones para la conservación de los bosques estacionalmente secos de Tarapoto, San Martín}

Los bosques estacionalmente secos de San Martín deben ser priorizados para la conservación por las siguientes razones:

Es el único lugar en el Perú donde algunas especies importantes de su flora pueden ser encontrados (p. e. Erythoxylum lucidum (Erythroxylaceae), Mosannona Raymondi (Annonaceae), Croton glabellus (Euphorbiaceae)).

Entre los bosques estacionalmente secos en Perú representan una muestra única debido a su localización aislada en el lado este de la región Andina, la cual es clave para entender el origen de estos bosques y la evolución de sus especies endémicas.

Debido a su cercanía a la carretera marginal de la Selva, estos hábitats son probablemente los más amenazados de su tipo en el Perú

Estos boques representan un complemento a los bosques tropicales más secos y mejor estudiados que ocurren en la región Pacífico del Perú (Tumbes y Piura) y en el valle del Marañón.

La presencia de estos bosques en San Martín ayudan a regular la dinámica del clima y la precipitación que afecta a muchos centros poblados del Huallaga Central incluyendo la ciudad de Tarapoto.
Las siguientes áreas deberían ser priorizadas en la conservación de los bosques estacionalmente secos de San Martín: el área al norte de Nueva Unión, el área al Sur de Picota, en la cuenca del Bombonajillo y Ponasillo y hacia el Sur de Ledoy. Estas áreas están bajo presión para la expansión de cultivos y la explotación maderera pero aun conservan sectores de bosques estacionalmente secos. Así mismo, estas áreas incluyen un gradiente de precipitación y humedad que se refleja en la abundancia de algunas de las especies arbóreas más representativas de estos bosques (M. bidentata, Myrcia sp. 3). El área de conservación municipal El Quinillal, creada por la Municipalidad de Picota, representa una importante iniciativa en el cual deberían basarse los futuros esfuerzos para la conservación de los bosques estacionalmente secos de Tarapoto.

\section{Agradecimientos}

Agradezco a Nicolás Flores Torres, Bedmar García Vela, Yisela Quispe Flores, Stephan Ramírez Ferry y Victor Chuquibala Montenegro por su invalorable apoyo en el campo. A Carlos Gonzáles y todo el personal del complejo turístico "Puerto Palmeras" - Tarapoto, que proporcionaron una estancia agradable durante el trabajo de campo en Juan Guerra y Yacucatina. Marco León y Reynaldo Linares-Palomino compartieron su entusiasmo en la conservación de los bosques secos de Tarapoto y dieron muy buenas sugerencias para el trabajo de campo. Angelito Paredes y Loiso Tello en Picota proporcionaron ayuda crítica para el trabajo de campo en la cuenca del río Bombonajillo, Picota. Gracias a Joaquina Alban y Hamilton Beltrán por ayudar amablemente durante el trabajo en el herbario San Marcos (USM) del Museo de Historia Natural en Lima. Paul Berry, Douglas Daly y Toby Pennington ayudaron en su especialidad taxonómica durante la fase de identificación. Gracias a Karla Meza por proporcionar comentarios útiles en un primer borrador de este artículo. José Alvarez Alonso, Filomeno Encarnación, Noam Shany y Byron Swift proporcionaron apoyo y sugerencias valiosas en varias fases del estudio. Este estudio fue financiado por Nature and Culture Internacional (NCI) con fondos adicionales de WorldParks.

\section{Literatura citada}

Bridgewater S., R.T. Pennington, C.A. Reynel, A. Daza \& T.D. Pennington. 2003. A preliminary floristic and phytogeographic analysis of the woody flora of seasonally dry forests in northern Peru. Candollea 58(1):129-148.

Caetano S., D.R. Prado, R.T. Pennington, et al. 2008. The history of seasonally dry tropical forests in Eastern South America: inferences from the genetic structure of the tree Astronium urundeuva (Anacardiaceae). Molecular Eecology 17: 3147-3159.

Condit R., R.B. Foster, S.P. Hubbell, et al. 1998. Assessing forest diversity on small plots: calibration using species-individual curves from 50 ha plots. Pages 247-268 in: Dallmeier, F. y J. A. Comiskey (eds.) Forest Biodiversity Research, Monitoring and Modeling. MAB Series Vol. 20, UNESCO, Paris, and Parthenon Publishing Group, N.Y.

Curtis J.T. \& R.P. McIntosh. 1951. An Upland forest continuum in the prairie-Forest border region of Wisconsin. Ecology 32(3): 476-496.

Encarnación F. 2004. Vegetación. En: Zonificación Ecológica Económica de la Región San Martín. Gobierno Regional de San Martín, Instituto de Investigaciones de la Amazonía Peruana (IIAP). 81 pp.

Fernández R. \& R. Vail. 2003. New records of Platycerium andinum Baker in Peru. American Fern Journal 93(3): 160-163. 
Foster R., H. Beltrán, \& W.S. Alverson. 2001. Flora y vegetación. Pages 50-64 in W. S. Alverson, L. O. Rodríguez, and D. K. Moskovits, editors. Perú: Biabo Cordillera Azul. The Field Museum, Chicago, IL.

Gentry A.H. 1995. Diversity and floristic composition of Neotropical dry forests. In: Seasonally Dry Tropical Forests. S. H. Bullock, H. H. Mooney, y E. Medina (eds.). Cambridge University Press. Pp. 146-194.

Holdridge L. 1967. Life Zone Ecology. Tropical Science Center, San José, Costa Rica.

INRENA. 1995. Mapa ecológico del Perú: guía explicativa. Ministerio de Agricultura, Instituto Nacional de Recursos Naturales (INRENA). Lima.

Janzen D.H. 1988. Tropical dry forests: the most endangered major tropical ecosystem. In: Biodiversity, E. O. Wilson (ed.). National Academy Press, Washington, D. C. pp. 130137.

Leal-Pinedo J.M. \& R. Linares-Palomino. 2005. Los bosques secos de la reserva de la biosfera del noroeste (Perú): diversidad arbórea y estado de conservación. Caldasia 27(2): 195-211.

León B. \& C. Monsalve. 2006. Erythroxylaceae endémicas del Perú. In: B. León et al. (Eds). El libro rojo de las plantas endémicas del Perú. Rev. peru. biol. Número especial 13(2): $294 \mathrm{~s}$

Linares-Palomino R. 2002. A floristic and phytogeographic analysis of Peruvian seasonally dry tropical forests. MSc thesis. University of Edinburgh, UK. 112 pp.

Linares-Palomino R. 2005. (en línea). Annotated Checklist of the woody plants in Peruvian seasonallyly dry forests. $<$ rbg-web2.rbge.org.uk/dryforest/database.htm>. Acceso 28/8/2008.

Linares-Palomino R. 2006. Phytogeography and Floristics of Seasonally Dry Tropical Forests in Peru. In: R.T. Pennington, G.P. Lewis, J.A. Ratter, eds. Neotropical Savannas and Seasonally Dry Forests: Plant Diversity, Biogeography, and Conservation, The Systematics Association Special Volume Series 69, Taylor and Francis Group. Pp. 257-280.
Linares-Palomino R. 2007. Lista anotada de plantas leñosas en bosques estacionalmente secos del Peru - una nueva herramienta en Internet para estudios taxonómicos, ecológicos y de biodiversidad. Arnaldoa 14(1): 149-152.

Linares-Palomino R. \& S.I. Ponce Álvarez. 2005. Tree community patterns in seasonally dry tropical forests in the Cerros de Amotape Cordillera, Tumbes, Peru. Forest Ecology and Management 209(3): 261-272.

Magurran A. E. 1988. Ecological diversity and its measurement. Princeton Univ. Press.

Maass J.M., H.D. Mooney, \& E. Medina. 1995. Conversion of tropical dry forest to pasture and agriculture. Pages 399-422 in S. H. Bullock, and editors. Seasonally Dry Tropical Forests. Cambridge University Press, New York.

McCune B. \& J. B. Grace. 2002. Analysis of ecological communities. MjM software design. USA. 300 pp.

Miles L., A.C. Newton, R.S. DeFries, et al. 2006. A global overview of the conservation status of tropical dry forests. Journal of Biogeography 33 (3): 491-505.

Ministerio de Agricultura. 2006. (en línea). Política agraria, plan estratégico regional San Martín. <www.minag.gob.pe/ polt_sanmartin2.shtml >. Acceso 20/6/2006.

Murphy P.G. \& A.E. Lugo. 1986. Ecology of tropical dry forest. Annual Review of Ecology and Systematics 17: 67-88.

ONERN. 1976. Mapa ecológico del Perú: Guía explicativa. Oficina Nacional de Evaluación de Recursos Naturales. Lima, Perú. 146 pp.

Phillips O.L. \& Miller J. 2004. Alwyn H. Gentry Forest Transect Data Set. Miss. Bot. Garden. Monographs, Missouri.

Prado D.E. \& P.E. Gibbs. 1993. Patterns of species distribution in the dry seasonal forests of South America. Annals of the Missouri Botanical Garden 80:902-927.

Trejo I. \& R. Dirzo. 2002. Floristic diversity of Mexican seasonally dry tropical forests. Biodiversity and Conservation 11: 2063-2084.

Apéndice 1. Índice de Valor de Importancia (IVI) de las especies encontradas en los bosques estacionalmente secos de Tarapoto. San Martín. Coll. № (número de colección representativo depositado en el herbario USM de la Universidad de San Marcos. Ade (Densidad absoluta). Rde (Densidad relativa). Ado (Dominancia absoluta). Rdo (Dominancia relativa). Af (Frecuencia absoluta). Rf (Frecuencia relativa). IVI (Índice de Valor de Importancia $=$ Rde + Rdo + Rf)

\begin{tabular}{|c|c|c|c|c|c|c|c|c|c|}
\hline Especie & Coll. $\mathbf{N}^{\circ}$ & Familia & Ade & Rde & Ado & Rdo & Af & $\mathbf{R f}$ & IVI \\
\hline Manilkara bidentata (A. DC.) A. Chev. & 4240 & Sapotaceae & 65 & 2,31 & 62373,88 & 27,71 & 9 & 1,92 & 31,94 \\
\hline Oxandra espintana (Spruce ex Benth.) Baill. & 4154 & Annonaceae & 296 & 10,52 & 17617,90 & 7,83 & 10 & 2,13 & 20,48 \\
\hline Trichilia sp. 1 & 4162 & Meliaceae & 88 & 3,13 & 8572,67 & 3,81 & 8 & 1,71 & 8,64 \\
\hline Brosimum sp. 1 & 4230 & Moraceae & 48 & 1,71 & 10215,32 & 4,54 & 9 & 1,92 & 8,16 \\
\hline Maytenus macrocarpa (Ruiz \& Pav.) Briq. & 4163 & Celastraceae & 99 & 3,52 & 5167,79 & 2,30 & 9 & 1,92 & 7,73 \\
\hline Myrcia sp. 1 & 4231 & Myrtaceae & 153 & 5,44 & 1931,71 & 0,86 & 5 & 1,07 & 7,36 \\
\hline Simira rubescens cf. & 4153 & Rubiaceae & 75 & 2,67 & 4821,91 & 2,14 & 8 & 1,71 & 6,51 \\
\hline Myrcia sp. 4 & 4215 & Myrtaceae & 101 & 3,59 & 2025,45 & 0,90 & 9 & 1,92 & 6,41 \\
\hline Myrcia sp. 3 & 4234 & Myrtaceae & 37 & 1,31 & 9017,15 & 4,01 & 4 & 0,85 & 6,17 \\
\hline Xylopia cuspidate Diels & 4166 & Annonaceae & 109 & 3,87 & 1500,29 & 0,67 & 7 & 1,49 & 6,03 \\
\hline Gustavia elliptica cf. & 4474 & Lecythidaceae & 96 & 3,41 & 3892,36 & 1,73 & 4 & 0,85 & 5,99 \\
\hline Capparis petiolaris Kunth & 4156 & Capparidaceae & 78 & 2,77 & 2325,20 & 1,03 & 9 & 1,92 & 5,72 \\
\hline Coccoloba sp. 3 & 4282 & Polygonaceae & 82 & 2,91 & 2807,62 & 1,25 & 7 & 1,49 & 5,65 \\
\hline Drypetes amazonica Steyerm. & 4164 & Euphorbiaceae & 50 & 1,78 & 4720,39 & 2,10 & 8 & 1,71 & 5,58 \\
\hline Capparis mollis Kunth & 4152 & Capparidaceae & 42 & 1,49 & 3861,85 & 1,72 & 8 & 1,71 & 4,91 \\
\hline Indet. 4 & 4192 & Indet. 4 & 52 & 1,85 & 3799,41 & 1,69 & 6 & 1,28 & 4,81 \\
\hline Cordia alliodora (Ruiz \& Pav.) Oken & 4150 & Boraginaceae & 55 & 1,95 & 1918,41 & 0,85 & 9 & 1,92 & 4,73 \\
\hline Coccoloba sp. 1 & 4202 & Polygonaceae & 34 & 1,21 & 2551,55 & 1,13 & 10 & 2,13 & 4,47 \\
\hline Zygia coccinea aff. & 4430 & Fabaceae & 73 & 2,59 & 2656,16 & 1,18 & 3 & 0,64 & 4,41 \\
\hline Schinopsis peruviana Engl. & 4151 & Anacardiaceae & 16 & 0,57 & 6706,15 & 2,98 & 4 & 0,85 & 4,40 \\
\hline Syagrus sancona H. Karst. & & Arecaceae & 24 & 0,85 & 3062,75 & 1,36 & 8 & 1,71 & 3,92 \\
\hline Pouteria sp. 1 & 4402 & Sapotaceae & 40 & 1,42 & 3293,71 & 1,46 & 4 & 0,85 & 3,74 \\
\hline
\end{tabular}


Apéndice 1. Conituación

\begin{tabular}{|c|c|c|c|c|c|c|c|c|c|}
\hline Especie & Coll. $\mathbf{N}^{\circ}$ & Familia & Ade & Rde & Ado & Rdo & Af & Rf & IVI \\
\hline Allophylus loretensis cf. & 4158 & Sapindaceae & 55 & 1,95 & 520,12 & 0,23 & 7 & 1,49 & 3,68 \\
\hline Alseis peruviana Standl. & 4172 & Rubiaceae & 24 & 0,85 & 2834,64 & 1,26 & 7 & 1,49 & 3,60 \\
\hline Erythroxylum lucidum Kunth & 4593 & Erythroxylaceae & 55 & 1,95 & 1486,16 & 0,66 & 4 & 0,85 & 3,47 \\
\hline Erythroxylum shatona J.F. Macbr. & 4157 & Erythroxylaceae & 41 & 1,46 & 676,59 & 0,30 & 8 & 1,71 & 3,46 \\
\hline Triplaris Americana L. & 4159 & Polygonaceae & 47 & 1,67 & 1180,27 & 0,52 & 5 & 1,07 & 3,26 \\
\hline Sorocea trophoides W.C. Burger & 4167 & Moraceae & 33 & 1,17 & 1290,39 & 0,57 & 7 & 1,49 & 3,24 \\
\hline Fabaceae sp. 1 & 4265 & Fabaceae & 25 & 0,89 & 1737,39 & 0,77 & 7 & 1,49 & 3,15 \\
\hline Protium puncticulatum J.F. Macbr. & 4403 & Burseraceae & 36 & 1,28 & 1124,98 & 0,50 & 6 & 1,28 & 3,06 \\
\hline Myrcia sp. 2 & 4214 & Myrtaceae & 25 & 0,89 & 2721,23 & 1,21 & 4 & 0,85 & 2,95 \\
\hline Trichilia rubra C. DC. & 4262 & Meliaceae & 33 & 1,17 & 1317,89 & 0,59 & 5 & 1,07 & 2,82 \\
\hline Mosannona raimondi (Diels) Chatrou & 4316 & Annonaceae & 35 & 1,24 & 1516,47 & 0,67 & 4 & 0,85 & 2,77 \\
\hline Ocotea sp. 1 & 4325 & Lauraceae & 25 & 0,89 & 1329,56 & 0,59 & 6 & 1,28 & 2,76 \\
\hline Inga cordatoalata Ducke & 4161 & Fabaceae & 25 & 0,89 & 811,62 & 0,36 & 7 & 1,49 & 2,74 \\
\hline Myrtaceae sp. 6 & 4260 & Myrtaceae & 36 & 1,28 & 1803,56 & 0,80 & 2 & 0,43 & 2,51 \\
\hline Euphorbiaceae sp. 1 & 4350 & Euphorbiaceae & 17 & 0,60 & 1880,13 & 0,84 & 5 & 1,07 & 2,51 \\
\hline Fabaceae sp. 6 & 4301 & Fabaceae & 6 & 0,21 & 3071,98 & 1,36 & 4 & 0,85 & 2,43 \\
\hline Indet. 2 & 4294 & Indet. 2 & 19 & 0,68 & 943,66 & 0,42 & 6 & 1,28 & 2,37 \\
\hline Trichilia maynasiana C. DC. ssp. Maynasiana & 4367 & Meliaceae & 34 & 1,21 & 983,31 & 0,44 & 3 & 0,64 & 2,28 \\
\hline Casearia sylvestris Sw. & 4200 & Flacourtiaceae & 18 & 0,64 & 463,06 & 0,21 & 6 & 1,28 & 2,12 \\
\hline Pouteria sp. 2 & 4538 & Sapotaceae & 27 & 0,96 & 1144,70 & 0,51 & 3 & 0,64 & 2,11 \\
\hline Randia armata (Sw.) DC. & 4643 & Rubiaceae & 15 & 0,53 & 639,83 & 0,28 & 6 & 1,28 & 2,10 \\
\hline Fabaceae sp. 3 & 4229 & Fabaceae & 15 & 0,53 & 1085,22 & 0,48 & 5 & 1,07 & 2,08 \\
\hline Urera baccifera (L) Gaudich. ex Wedd. & 4209 & Urticaceae & 14 & 0,50 & 601,36 & 0,27 & 6 & 1,28 & 2,04 \\
\hline Trichilia elegans A. Juss. & 4182 & Meliaceae & 15 & 0,53 & 901,69 & 0,40 & 5 & 1,07 & 2,00 \\
\hline Coccoloba sp. 2 & 4203 & Polygonaceae & 17 & 0,60 & 1667,99 & 0,74 & 3 & 0,64 & 1,98 \\
\hline Myrcia sp. 5 & 4300 & Myrtaceae & 28 & 1,00 & 412,23 & 0,18 & 3 & 0,64 & 1,82 \\
\hline Aspidosperma sp. 1 & 4165 & Apocynaceae & 7 & 0,25 & 2066,17 & 0,92 & 3 & 0,64 & 1,81 \\
\hline Rollinia mucosa (Jacq.) Baill. & 4578 & Annonaceae & 12 & 0,43 & 558,50 & 0,25 & 5 & 1,07 & 1,74 \\
\hline Cathedra sp. 1 & 4264 & Olacaceae & 14 & 0,50 & 805,03 & 0,36 & 4 & 0,85 & 1,71 \\
\hline Ceiba insignis (kunth) P.E. Gibbs \& Semir & 4598 & Bombacaceae & 7 & 0,25 & 2203,98 & 0,98 & 2 & 0,43 & 1,65 \\
\hline Tabebuia billbergii (Bureau \& K. Schum.) Standl. & 4343 & Bignoniaceae & 11 & 0,39 & 866,93 & 0,39 & 4 & 0,85 & 1,63 \\
\hline Platymiscium sp. 1 & 4277 & Fabaceae & 15 & 0,53 & 504,47 & 0,22 & 4 & 0,85 & 1,61 \\
\hline Alibertia edulis (Rich.) A. Rich. ex DC. & 4195 & Rubiaceae & 10 & 0,36 & 264,93 & 0,12 & 5 & 1,07 & 1,54 \\
\hline Neea sp. 4 & 4207 & Nyctaginaceae & 12 & 0,43 & 581,23 & 0,26 & 4 & 0,85 & 1,54 \\
\hline Aspidosperma capitatum aff. & 4320 & Apocynaceae & 5 & 0,18 & 1098,74 & 0,49 & 4 & 0,85 & 1,52 \\
\hline Ocotea cernua (Nees) Mez & 4204 & Lauraceae & 8 & 0,28 & 1767,14 & 0,78 & 2 & 0,43 & 1,50 \\
\hline Croton glabellus L. & 4363 & Euphorbiaceae & 17 & 0,60 & 428,44 & 0,19 & 3 & 0,64 & 1,43 \\
\hline Pachira aquatica Aubl. & 4645 & Bombacaceae & 3 & 0,11 & 1459,45 & 0,65 & 3 & 0,64 & 1,39 \\
\hline Pouteria subrotata cf. & 4389 & Sapotaceae & 5 & 0,18 & 2160,76 & 0,96 & 1 & 0,21 & 1,35 \\
\hline Triplaris sp. 1 & 4395 & Polygonaceae & 12 & 0,43 & 95,29 & 0,04 & 4 & 0,85 & 1,32 \\
\hline Ocotea bofo & 4224 & Lauraceae & 12 & 0,43 & 463,46 & 0,21 & 3 & 0,64 & 1,27 \\
\hline Myrtaceae sp. 4 & 4582 & Myrtaceae & 7 & 0,25 & 363,38 & 0,16 & 4 & 0,85 & 1,26 \\
\hline Croton sp. 1 & 4486 & Euphorbiaceae & 19 & 0,68 & 345,72 & 0,15 & 2 & 0,43 & 1,26 \\
\hline Myrtaceae sp. 9 & 4635 & Myrtaceae & 13 & 0,46 & 144,36 & 0,06 & 3 & 0,64 & 1,17 \\
\hline Zanthoxylum acreanum aff. & 4347 & Rutaceae & 4 & 0,14 & 820,39 & 0,36 & 3 & 0,64 & 1,15 \\
\hline Pouteria sp. 3 & 4426 & Sapotaceae & 7 & 0,25 & 53,07 & 0,02 & 4 & 0,85 & 1,13 \\
\hline Neea sp. 2 & 4642 & Nyctaginaceae & 4 & 0,14 & 291,07 & 0,13 & 4 & 0,85 & 1,12 \\
\hline Fabaceae sp. 7 & 4279 & Fabaceae & 3 & 0,11 & 822,53 & 0,37 & 3 & 0,64 & 1,11 \\
\hline Chrysophyllum sp. 1 & 4220 & Sapotaceae & 8 & 0,28 & 330,57 & 0,15 & 3 & 0,64 & 1,07 \\
\hline Bunchosia angustifolia cf. & 4187 & Malpighiaceae & 5 & 0,18 & 186,46 & 0,08 & 3 & 0,64 & 0,90 \\
\hline Erythroxylum fimbriatum Peyr. & 4268 & Erythroxylaceae & 6 & 0,21 & 84,09 & 0,04 & 3 & 0,64 & 0,89 \\
\hline Indet. 1 & 4271 & Indet. 1 & 4 & 0,14 & 663,67 & 0,29 & 2 & 0,43 & 0,86 \\
\hline Arecaceae sp. 1 & 4599 & Arecaceae & 11 & 0,39 & 510,16 & 0,23 & 1 & 0,21 & 0,83 \\
\hline Phytecellobium sp. 1 & 4443 & Fabaceae & 7 & 0,25 & 198,57 & 0,09 & 2 & 0,43 & 0,76 \\
\hline Leucaena leucocephala (Lam.) de Wit. & 4284 & Fabaceae & 4 & 0,14 & 407,04 & 0,18 & 2 & 0,43 & 0,75 \\
\hline Sapium marmieri aff. & 4408 & Euphorbiaceae & 2 & 0,07 & 450,83 & 0,20 & 2 & 0,43 & 0,70 \\
\hline Ouratea tarapotensis J.F. Macbr. & 4304 & Ochnaceae & 6 & 0,21 & 56,59 & 0,03 & 2 & 0,43 & 0,66 \\
\hline Casearia pitumba Sleumer & 4336 & Flacourtiaceae & 4 & 0,14 & 166,09 & 0,07 & 2 & 0,43 & 0,64 \\
\hline Brosimum alicastrum $\mathrm{Sw}$. & 4191 & Moraceae & 3 & 0,11 & 211,38 & 0,09 & 2 & 0,43 & 0,63 \\
\hline Myrtaceae sp. 8 & 4636 & Myrtaceae & 4 & 0,14 & 114,67 & 0,05 & 2 & 0,43 & 0,62 \\
\hline Pouteria sp. 5 & 4393 & Sapotaceae & 2 & 0,07 & 232,72 & 0,10 & 2 & 0,43 & 0,60 \\
\hline Steriphoma peruvianum Spruce ex Eichler & 4322 & Capparidaceae & 4 & 0,14 & 64,68 & 0,03 & 2 & 0,43 & 0,60 \\
\hline Pouteria bangii (Rusby) T.D. Penn. & 4625 & Sapotaceae & 4 & 0,14 & 64,36 & 0,03 & 2 & 0,43 & 0,60 \\
\hline Myrtaceae sp. 1 & 4160 & Myrtaceae & 4 & 0,14 & 20,00 & 0,01 & 2 & 0,43 & 0,58 \\
\hline Pisonia sp. 1 & 4438 & Rubiaceae & 3 & 0,11 & 37,56 & 0,02 & 2 & 0,43 & 0,55 \\
\hline Indet. 3 & 4428 & Indet. 3 & 3 & 0,11 & 24,28 & 0,01 & 2 & 0,43 & 0,54 \\
\hline Solanum appressum K.E. Roe & 4208 & Solanaceae & 2 & 0,07 & 91,96 & 0,04 & 2 & 0,43 & 0,54 \\
\hline Neea sp. 1 & 4354 & Nyctaginaceae & 2 & 0,07 & 82,57 & 0,04 & 2 & 0,43 & 0,53 \\
\hline Ampelocera edentula Kuhlm. & 4237 & Ulmaceae & 2 & 0,07 & 522,58 & 0,23 & 1 & 0,21 & 0,52 \\
\hline
\end{tabular}


Apéndice 1. Conituación

\begin{tabular}{|c|c|c|c|c|c|c|c|c|c|}
\hline Especie & Coll. $\mathbf{N}^{\circ}$ & Familia & Ade & Rde & Ado & Rdo & Af & Rf & IVI \\
\hline Cactaceae sp. 2 & 4285 & Cactaceae & 2 & 0,07 & 37,20 & 0,02 & 2 & 0,43 & 0,51 \\
\hline Neea sp. 3 & 4330 & Nyctaginaceae & 2 & 0,07 & 28,39 & 0,01 & 2 & 0,43 & 0,51 \\
\hline Trichilia pallida Sw. & 4328 & Meliaceae & 2 & 0,07 & 22,91 & 0,01 & 2 & 0,43 & 0,51 \\
\hline Astronium sp. 1 & 4390 & Anacardiaceae & 2 & 0,07 & 20,52 & 0,01 & 2 & 0,43 & 0,51 \\
\hline Talisia macrophylla (Mart.) Radlk. & 4315 & Sapindaceae & 2 & 0,07 & 14,41 & 0,01 & 2 & 0,43 & 0,50 \\
\hline Crematosperma sp. 1 & 4392 & Annonaceae & 6 & 0,21 & 171,62 & 0,08 & 1 & 0,21 & 0,50 \\
\hline Fabaceae sp. 8 & 4299 & Fabaceae & 2 & 0,07 & 433,94 & 0,19 & 1 & 0,21 & 0,48 \\
\hline Fabaceae sp. 5 & 4188 & Fabaceae & 2 & 0,07 & 264,08 & 0,12 & 1 & 0,21 & 0,40 \\
\hline Clusia sp. 2 & 4283 & Clusiaceae & 2 & 0,07 & 247,45 & 0,11 & 1 & 0,21 & 0,39 \\
\hline Esenbeckia sp. 1 & 4280 & Rutaceae & 4 & 0,14 & 79,21 & 0,04 & 1 & 0,21 & 0,39 \\
\hline Flacourtiaceae sp. 1 & 4482 & Flacourtiaceae & 1 & 0,04 & 262,19 & 0,12 & 1 & 0,21 & 0,37 \\
\hline Tabebuia sp. 1 & 4602 & Bignoniaceae & 3 & 0,11 & 73,13 & 0,03 & 1 & 0,21 & 0,35 \\
\hline Clusia sp. 1 & 4608 & Clusiaceae & 2 & 0,07 & 125,25 & 0,06 & 1 & 0,21 & 0,34 \\
\hline Agonandra peruviana aff. & 4196 & Opiliaceae & 1 & 0,04 & 201,34 & 0,09 & 1 & 0,21 & 0,34 \\
\hline Clavija sp. 1 & 4404 & Theophrastaceae & 3 & 0,11 & 40,87 & 0,02 & 1 & 0,21 & 0,34 \\
\hline Rhamnidium elaeocarpum Reissek & 4631 & Rhamnaceae & 2 & 0,07 & 117,93 & 0,05 & 1 & 0,21 & 0,34 \\
\hline Citharexylum sp. 1 & 4373 & Verbenaceae & 1 & 0,04 & 183,35 & 0,08 & 1 & 0,21 & 0,33 \\
\hline Indet. 5 & 4183 & Indet. 5 & 2 & 0,07 & 48,63 & 0,02 & 1 & 0,21 & 0,31 \\
\hline Luehea paniculata Mart. & 4289 & Tiliaceae & 1 & 0,04 & 110,12 & 0,05 & 1 & 0,21 & 0,30 \\
\hline Capparis amplissima Lam. & 4440 & Capparidaceae & 2 & 0,07 & 28,79 & 0,01 & 1 & 0,21 & 0,30 \\
\hline Guettarda aromatica Poepp. \& endl. & 4352 & Rubiaceae & 2 & 0,07 & 27,70 & 0,01 & 1 & 0,21 & 0,30 \\
\hline Psychotria sp. 1 & 4584 & Rubiaceae & 2 & 0,07 & 26,38 & 0,01 & 1 & 0,21 & 0,30 \\
\hline Tabernaemontana arcuata Ruiz \& Pav. & 4329 & Apocynaceae & 2 & 0,07 & 24,78 & 0,01 & 1 & 0,21 & 0,30 \\
\hline Garcinia macrophylla Mart. & 4257 & Clusiaceae & 2 & 0,07 & 23,76 & 0,01 & 1 & 0,21 & 0,29 \\
\hline Terminalia sp. 1 & 4185 & Combretaceae & 1 & 0,04 & 102,56 & 0,05 & 1 & 0,21 & 0,29 \\
\hline Brosimum guianense (Aubl.) Huber & 4600 & Moraceae & 1 & 0,04 & 101,99 & 0,05 & 1 & 0,21 & 0,29 \\
\hline Cymbopetalum longipes Benth. ex Diels & 4361 & Annonaceae & 2 & 0,07 & 12,20 & 0,01 & 1 & 0,21 & 0,29 \\
\hline Tabebuia chrysantha (Jacq.) G. Nicholson & 4295 & Bignoniaceae & 1 & 0,04 & 71,62 & 0,03 & 1 & 0,21 & 0,28 \\
\hline Maytenus sp. 1 & 4531 & Celastraceae & 1 & 0,04 & 52,15 & 0,02 & 1 & 0,21 & 0,27 \\
\hline Randia ruiziana DC. & 4293 & Rubiaceae & 1 & 0,04 & 49,74 & 0,02 & 1 & 0,21 & 0,27 \\
\hline Indet. 6 & 4334 & Indet. 6 & 1 & 0,04 & 41,01 & 0,02 & 1 & 0,21 & 0,27 \\
\hline Heisteria acuminata (Humb. \& Bonpl.) Engl. & 4401 & Olacaceae & 1 & 0,04 & 39,22 & 0,02 & 1 & 0,21 & 0,27 \\
\hline Cactaceae sp. 1 & 4348 & Cactaceae & 1 & 0,04 & 32,15 & 0,01 & 1 & 0,21 & 0,26 \\
\hline Cecropia polystachya Trécul & 4477 & Cecropiaceae & 1 & 0,04 & 31,20 & 0,01 & 1 & 0,21 & 0,26 \\
\hline Myrtaceae sp. 7 & 4391 & Myrtaceae & 1 & 0,04 & 26,07 & 0,01 & 1 & 0,21 & 0,26 \\
\hline Malpighiaceae sp. 1 & 4281 & Malpighiaceae & 1 & 0,04 & 25,21 & 0,01 & 1 & 0,21 & 0,26 \\
\hline Diospyros arthantifolia $c f$. & 4255 & Ebenaceae & 1 & 0,04 & 23,27 & 0,01 & 1 & 0,21 & 0,26 \\
\hline Chrysophyllum venezuelanense aff. & 4491 & Sapotaceae & 1 & 0,04 & 22,46 & 0,01 & 1 & 0,21 & 0,26 \\
\hline Apocynaceae sp. 1 & 4398 & Apocynaceae & 1 & 0,04 & 21,93 & 0,01 & 1 & 0,21 & 0,26 \\
\hline Myrtaceae sp. 5 & 4228 & Myrtaceae & 1 & 0,04 & 20,88 & 0,01 & 1 & 0,21 & 0,26 \\
\hline Micropholis egensis (A. DC.) Pierre & 4557 & Sapotaceae & 1 & 0,04 & 19,87 & 0,01 & 1 & 0,21 & 0,26 \\
\hline Rubiaceae sp. 1 & 4327 & Rubiaceae & 1 & 0,04 & 16,05 & 0,01 & 1 & 0,21 & 0,26 \\
\hline Myrtaceae sp. 3 & 4276 & Myrtaceae & 1 & 0,04 & 16,05 & 0,01 & 1 & 0,21 & 0,26 \\
\hline Nyctaginaceae sp. 1 & 4263 & Nyctaginaceae & 1 & 0,04 & 15,60 & 0,01 & 1 & 0,21 & 0,26 \\
\hline Annona sp. 1 & 4227 & Annonaceae & 1 & 0,04 & 15,38 & 0,01 & 1 & 0,21 & 0,26 \\
\hline Ficus caballina Standl. & 4444 & Moraceae & 1 & 0,04 & 14,29 & 0,01 & 1 & 0,21 & 0,26 \\
\hline Allophylus floribundus (Poepp.) Radlk. & 4639 & Sapindaceae & 1 & 0,04 & 8,94 & 0,00 & 1 & 0,21 & 0,25 \\
\hline Garcinia madruno cf. & 4394 & Clusiaceae & 1 & 0,04 & 8,44 & 0,00 & 1 & 0,21 & 0,25 \\
\hline Mayna odorata Aubl. & 4605 & Flacourtiaceae & 1 & 0,04 & 7,64 & 0,00 & 1 & 0,21 & 0,25 \\
\hline Tabernaemontana cymosa Jacq. & 4396 & Apocynaceae & 1 & 0,04 & 7,33 & 0,00 & 1 & 0,21 & 0,25 \\
\hline Morisonia oblongifolia Britton & 4481 & Capparidaceae & 1 & 0,04 & 6,45 & 0,00 & 1 & 0,21 & 0,25 \\
\hline Coccoloba sp. 4 & 4473 & Polygonaceae & 1 & 0,04 & 5,35 & 0,00 & 1 & 0,21 & 0,25 \\
\hline Psychotria viridis Ruiz \& Pav. & 4345 & Rubiaceae & 1 & 0,04 & 5,09 & 0,00 & 1 & 0,21 & 0,25 \\
\hline Myrtaceae sp. 2 & 4205 & Myrtaceae & 1 & 0,04 & 5,09 & 0,00 & 1 & 0,21 & 0,25 \\
\hline Fabaceae sp. 4 & 4429 & Fabaceae & 1 & 0,04 & 5,09 & 0,00 & 1 & 0,21 & 0,25 \\
\hline Total & & & 2814 & 100,00 & 225115,80 & 100,00 & 469 & 100,00 & 300,00 \\
\hline
\end{tabular}

